\title{
Scheidenverletzung bei der Spontangeburt einer Multipara.
}

\author{
Von Dr. E. Vogt.
}

Zerreißungen der Scheide sind eine häufige Geburtsverletzung. Ihre Gefährlichkeit hängt vor allem von ihrer Lage ab. Die Risse, welche oberhalb des Diaphragma pelvis liegen, können durch übermäßige Dehnung der Vagina in der Längsoder Querrichtung entstehen.

Die durch Längsdehnung entstandenen Risse verlaufen quer, nach Berry Hart (1) Dehnungsrisse, nach Sänger Schlitzrisse in der Scheide. Diese Verletzungen beschränken sich entweder nur auf ein Scheidengewölbe oder sie verlaufen zirkulär. Die Scheide kann dann vollständig. von der Zervix abgetrennt werden, Kolporrhexis.

Die Längs- oder Spaltrisse der Vagina kommen durch Ueberdehnung in zirkulärer Richtung zustande, sie beginnen fast stets oberhalb der Mitte und gehen meist in infra-diaphragmatische über.

Fast immer ist es der Kopf, welcher als vorangehender Teil zu einer Ueberdehnung und Verletzung der Vagina führt. Besondere Begleitumstände sind da wichtig: zu stürmische Wehen, enge und rigide Scheide, sehr starrer Beckenboden, alte Narben, besondere Zerreißlichkeit des Gewebes.

Bei Spontangeburt, besonders bei Mehrgebärenden, sind Längsrisse im oberen Drittel allein selten. Nur im Fall von Daniel war es zu einer" isolierten Ruptur im oberen Drittel der Vagina bei einer Spontangeburt gekommen. Ueber einen ähnlichen Fall möchte ich kurz berichten:

Geburtsgeschichte: 11. Para, 10 Snontangeburten. Die letzte Periode Ende Oktober, wie gewöhnlich. Die äußere Untersuchung ergab: Beckenmaße: $24,26,30,19$. Erste Srhädellage, Kopf schwer beweglich im Beckeneingang, Herztöne links unterhalb des Nabels, 126, regelmäßig. Wehenbeginn 6. August $19119 \mathrm{Uhr}$ vormittags. Wehen ganz 
unregclmäßig, schwach, alle 4-6 Minuten. 3,40 Uhr nachmittags innere Untersuchung: Muttermind fünfmarkstückgro B, Kopf im Peckeneingang, Pfeilnaht im crsten schrägen Durchmesscr. Blase steht. Besken ohne Besondorheiten. 4,50 Uhr nachmittags springt die Blase, es entIcert sich wenig klares Fruchtwasser.

$5,05 \mathrm{tTh}$ nachmittags wurde der Kopf in der Tiefe sichtbar.

5,15 Uhr nachmittags erfolgte die Spontangeburt cines $50 \mathrm{~cm}$ langen, 2900 g schweren Mädchens, das sofort lebhaft schric.

Dic Besichtigung des Scheidencinganges und des Dammes post partum ließ leine Verlctrung nachweisen.

5,45 Uhr nachmittags wurde auf leichten Druck die gelöste Nachgeburt ausgcstoßen, Fruchtkuchen und Eihäutc waren vollständig.

In der Nachgeburtsperinde batte die Frau nicht mehr als gewöhnlich gebdntet. Obwohl die Gebärmutter iiberwacht u urde, ging etwas dunkles Blut ab, doch war der Uterus sehr gut kontrahicrt. Die Nachwehen waren kräftig. Aus der Gcbärmulter, d. h. von der Haftflichch des Fruchtkuc!lens, konnte die Blutung nicht stammen. Als bei weiterer Beobachtung sich die Blutung versthirkte, die Franl blaß wurde und der Puls auf 120 stieg, war es klar, daß es sich nur um eine Blutung aus einer versteckten. ticfcren Rißverletzung handeln konnte, daher wurde sofort zur Einstcllung geschritten. Dic Blutung konnto nur noch aus der 7.ervix, dem Muttermund oder aus dem Scheidenrohr stammen. Bei der Entfaltung der Vagina mit ganz breiten Rinnen zeigte sich im mittleren Drittel der Sthciłe rechts der Columna rugarum posterior eine weit klaffende Wunde mit z.crfetzten Rändern, die schräg nach oben zog, um sirh im oberen Drittel der Sclleide bis fast. an das Scheidengewölbe auf der rechten Seito fortzusetzen. Im Scheidengewölbe selhst zog dann die Wunde im Bogen von außen rcehts mehr nach der Mittellinie zu bis fast zur Ansatzstelle der Portio. Alus der Wunde quoll andruernd dunkles Blut. Bei der Entfaltung der Seheide ficl es auf, daß dic Venen der Submukosa varikös entartet waren, verschiedentlich waren großc, klaffende Gefäßlımina zu sehen.

Zur Vercinigung des Wunde und zur Blutstillung mußte eine größerc Reihe von Katgutknopfnäbten gelegt werden.

Nach der Naht dieser Wunde auf der reshten Seite stand aber dic Blutung nicht; jetzt kam das Blut von der linken Scitc her aus einer ähnlichen Vcrletzung wic rechts; hier vcrlief, im hinteren Drittel der Schcide beginnend, eine ungefähr $7 \mathrm{~cm}$ lange, klaffende Wunde zwischen der Columna rugarum posterior und der linken seitlichen Scheidenwand in die. Höhe, bis ungefähr einen Qnerfinger unterhalb des Po tioansatzes. Krst nachdem auch diese Wunde durch mehrere Katgutnähte versorgt war, stand die Blutung. Die ganzc Schcide wurde dann vorsichtig mit stcriler Gazc tamponiert; dic ziemlich beträchtliche Anämie der Frau wurde durch subkutanc Kochsalzinfusion und durch Kampferinjektionen bekämpft. Darauf erholte sich die Frau schnell.

Das Wochenbett war bis zum clften Tage fieberfrci. Plötzlich etieg die Temperatur auf $39^{\circ} \mathrm{C}$; der Puls war dabei stets unter 100.

Es hatte sich eine Parametritis ausgebildet. Dic entzündliche Sclıwellung in hinteren Scheidengewölbe ging zuriick, gleichzeitig kehrte die Temperatur zur Norm zarück, sodaß die Frau am siebzehnten Tage beschwerdefrei cntlassen werden konnte. Die Verletzung der Scheide war per primam geheilt.

Wenn man sich die Verletzungen zu erklären versucht, muß man zunächst den Geburtsverlauf berücksichtigen.

Aus dern geht nur hervor, daß die Geburt bei anscheinend gallz normalen Becken- und Weichteilsverhältnissen ohne jede Kunsthilfo verlief. Sicher bestand kein Mißverhältnis zwischen Frucht und Becken. Das Kind lag in erster Hinterhauptslage, dem entsprach auch der Geburts. mechanismis.

Die Größe des Kindes kommt dann auch in Frage.

Es war $50 \mathrm{~cm}$ lang, $2900 \mathrm{~g}$ schwer, der Kopf war nicht besonders hart und so gut wie garnicht konfiguriert, cin Beweis dafür, daß er schnell und, ohne ein Hindernis zu finden, durch den Geburtskanal hindurchgetreten war. Das Kind zeigte die Zeichen der Reife, es war wohlgebildet, besonders fehlte jede MiBbildung am Schädel.

Eine stürmische Wehentätigkeit, welche die Scheide zu plötzlich dehnt, war nicht beobachtet worden.

Offenbar lagen aber doch ganz besondere prädisponierende Momente vor.

Zunächst ist da wohl der allgemoine Ernährungszustand der Frau von Bedeutung. Ein krankhafter Befund an den inneren Organen ließ sich nicht erhcben, auch von Konstitutionskrankheitcn, wie Lues, Nephritis, Tuberkulcse, fand sich nichts, nur war der Ernährungszustand der Frau sehr dürftig. Das Fettpolster war schlecht entwickelt, die Gesich ts. farbe und die sichtbaren Schleimhäute bla $B$, die Gesichtszüge eingefallen, die Frau mußte stets während der ganzen Schwangerschaft schwer körporlich arbeiten.

Sodann bat die Frau in zwölf Jahren elf Schwangerschaften durchgemant, sodaß zweifellos eino mangelhafte Rückbildung dor Genitalien und allgemeine Unterernährung vorlag.
Vielleiclit war auch die Zerreißlichkeit des Gewcbes durch lokale Momente bedingt, entzündliche Prozesse, altc Narben, Mangel an elasti. sclen Fascrn. Es fehlte aber jede Spur eines Katarrhs oder oines Nooplasmas. Ein Hängebauch, bei dem gerade die hintere Vaginalwand beim Durchtritt des Kopfes besonders beansprucht wird, bestand nicht.

Der knöcherne Geburtskanal kann nicht in Frage kommen, die Beckenneigung war normal, der Schambogen weit, Exostosen fehlten. Für Hypoplasie oder Infantilismus der inneren Genitalion sprach nichts. Ob die variköse Entartung der Venen der Submukcsa der Scheide, wclche fast in kleinfingerdicke Gebilde umgewandelt waren, nicht die Haupt. clisposition anatomisch abgab, diese Vermutung liegt sehr nahe. Das lockere, weitmaschige, fetthaltige Bindegewebslager, in dem der slipradiapliragmatische Teil der Scleeide liegt, ist sehr reich an Venenplexus. Nur dadurch ist es möglich, daß sich die Scheide infolge ihrer greßen Verschieblichkeit dem Füllungszustande ihrer Nachbarorgane anpassen kann.

Zur feineren Anatomie der Vagina bildet unserer Fall einen klinischen Beitrag.

Die Risse verliefen seitlich. weil hier die Muskulatur am schwächsten entwickelt ist. Sic gingen nic.lıt ganz bis zum Scheidengewölbe, nur auf der rechten Seite; das kommt daher, daß die hintere Vaginalwand am Zcrvixansatz die dickste Muskulatur und größte Dickc besitzt, nach Wald c y c $0,3-0,4 \mathrm{~cm}$. Unterhalb des Scheidenansatzes liegt anatomisch die dünnste Stelle der Vagina. Sodanı liefen die Risse lateral derVerdickung, welche Waldoycr als Portiopolster beschrieb, und latcral der Columna rugarum posterior, welche in dcr Scheidenmitte beginnt.

Die Seitenwände der Vagina sind anatomisch für Längsrissc prädestiniert. Die Muskulatur ist hier-am dürftigsten entwickelt. Nach $\mathrm{J}$. Kocks wird die Columna rugarum posterior sclten in den $\mathrm{RiB}$ hireingozogen, weil hier das Gewebe straffer und dichter ist. Sodann ist die Elastizitait der hinteren Scheide gcringer als die der vorderen. Auch disponiert die hintere. Vaginalwand viel mehr zu Geburtstraumen, woil s:c sehr stark ausgedchnt werden muß, bevor sie in die Konkavität des Kreuzbcins eingedriickt wird, und hier an Knochen Stïtze und Scliutz. und ein Widerlager findet.

1)as Gesetz von J. Kocks (2), wonach die Stellung und Bewegungsrichtung des Kopfes die Seite des Risses bestimmt, oder die Regel von W. A. Freund (3): die Stirn bezeichnet die Iage und Richtung der Läsion, vorausgesetzt, daß die Scheide außer der Engigkeit keine komplizierenden Anomalien bietet, wird bestätigt. Der $\mathrm{Ri} B$ war rechts ausgedehnter und tiefer als links. Es handelte sich um eine erste Hinterhauptslage, Rücken vorn.

Die Blutung stammte aus den Venen des Plexus uterovaginalis, die varikös entartet waren. Die Hämorrhagic aus diesen Venen kann letal werden, trotz sofortiger Hilfe in der Klinik, wie in dem. Fall von Sietzenfrey (4).

Auch E. Ka uf man n sezierte eine Frau, die sich aus einem geplatzten Varix im Plexus uterovaginalis verblutet hatte.

Für die Behandlung solcher Risse kommt nur Bloßlegung mit breiten Rinnenspekula und Vereinigung durch die Naht in Frage, welche ja auch die sicherste Blutstillung gewährleiste.t. Tamponade ist nur als Notbehelf erlaubt, denn wenn die Blutung stehen soll, muß die Ausstopfung sehr fest sein, was wieder infolge starker Schmerzhaftigkeit ohne Narkose. unmöglich wird. Sodann kann man die Tamponade höchstens 24-48 Stunden liegen lassen; abgesehen von der Gefahr der Nachblutung, sofern jetzt nicht eine Sekundärnaht gelegt wird, droht dann die große Gefahr der Infektion, wenn die bakterienreichen Lochien über die Wunde flieben.

Prognostisch sind die schwereren Scheidenverletzungen, sofern keine Infektion eintritt, nicht ungünstig, wie unser Fall zeigt. Nur bei Eröffnung des Peritoneums ist die Gefahr der Peritonitis sehr groß. Wichtig ist, jede Staung der Lochien durch Hochlegung des Oberkörpers oder entsprechende Seitenlagerung $z u$ vermeiden. Nur in seltenen Fällen kommt es zu Verwachsungen, Stenose und Atresie der Vagina.

Auch für die gerichtsärztliche Geburtshilfe dürfte die Kenntnis derartiger seltener Komplikationen von Bedeutung sein.

Literatur: Berry Hart u. folgende, Siehe Handb. d. Geburtshilfe f. Oeburtsh. 56 